\title{
The dark side of p21
}

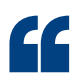

prolonged $\mathrm{p} 21$

expression in

p53-deficient

cells can

deregulate

DNA

replication
The cyclin-dependent kinase inhibitor p21 is a major effector of the tumour suppressor p53. It mediates cell cycle arrest in the G1 phase and cell senescence in response to several stimuli, including oncogene-induced proliferation. However, p21 can be activated independently of p53 and has cancer-promoting properties, such as supporting tumour growth in mice, the mechanisms of which are unclear. Galanos et al. now report that prolonged $\mathrm{p} 21$ expression in p53-deficient cells leads to deregulation of DNA replication and genomic instability.

When examining various p53-deficient human carcinomas and precancerous lesions, the authors found atypical cells that expressed both $\mathrm{p} 21$ and the proliferation marker Ki67. To study the

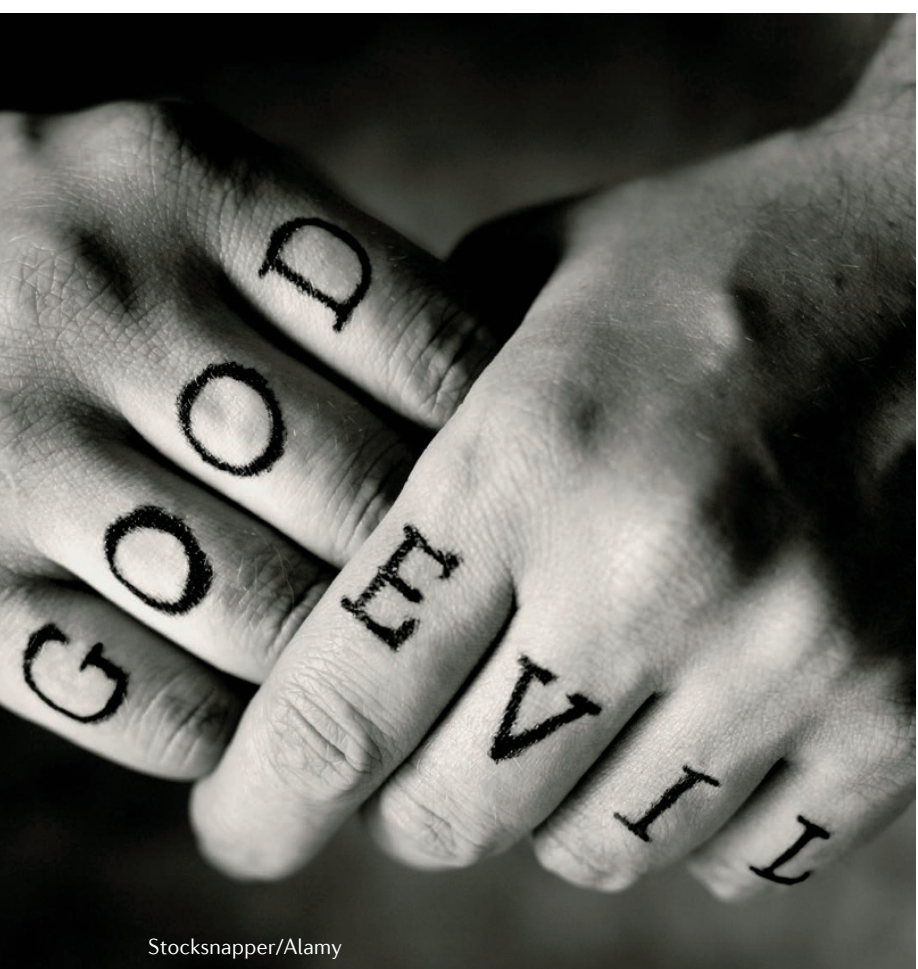

proliferative and other effects of p21 expression in a p53-null background, an inducible p21 module was introduced into two p53-null cell types - the osteosarcoma cell line Saos2 and precancerous Li-Fraumeni syndrome-derived fibroblasts. Following p21 induction, the growth rate of the cells was reduced and they accumulated in G1, despite an increase in the protein levels of DNA replication licensing factors, such as CDT1 and CDC6 (cell division control protein 6 homologue).

CDT1, CDC6 and p21 are all ubiquitylated by the E3-ubiquitin ligase complex CRL4-CDT2. Unlike wild-type p21, the expression of a p21 mutant that cannot be targeted for degradation by CRL4-CDT2 did not lead to higher levels of CDT1 and CDC6, and shutting-off wild-type p21 expression led to ubiquitylationdependent decrease of CDT1 levels. By contrast, induction of $\mathrm{p} 21$ expression in wild-type p53 cells resulted in considerable apoptosis and a decrease in CDT1 and CDC6 levels. These results indicate that, in the absence of p53, high p21 levels may saturate CRL4-CDT2 and lead to the accumulation of its other targets, including CDT1 and CDC6.

Inappropriate expression of replication licensing factors results in unscheduled replication (re-replication), replication stress and DNA damage. Expression of p21, but not of the CRL4-CDT2resistant p21 mutant, led to the accumulation of DNA damage and cells with doubled DNA content, which is indicative of re-replication; depletion of CDT1 and CDC6 abolished these effects. Inspection of replication intermediates by electron microscopy revealed that, upon p21 expression, Saos 2 cells decreased replication fork reversal (maintenance) and accumulated stretches of single-stranded DNA at replication forks. DNA damage accumulation following replication stress was dependent on the activity of the structure-specific resolvase complex MUS81-EME1, and damage repair was dependent on RAD52, which is involved in the error-prone microhomology-mediated repair.

Sustained p53-independent p21 expression in the Saos 2 and Li-Fraumeni syndrome cells triggered their senescence in a manner that was dependent on CDT1 and CDC6. However, 10 days after p21 induction, subpopulations of proliferating cells progressively emerged that expressed p21, Ki67, and CDC6 and/or CDT1. In these 'escaped cells', DNA damage was reduced through the activity of MUS81-EME1RAD52 and this was accompanied by the appearance of micronuclei, which indicates that the repair was errorprone. Consequently, the escaped cells exhibited various chromosomal aberrations and even chromosome shattering and reconstitution. Furthermore, the cells acquired enhanced anchorage-independent growth and invasiveness in vitro and higher tolerance to treatment with genotoxic anticancer drugs.

In summary, prolonged $\mathrm{p} 21$ expression in p53-deficient cells can deregulate DNA replication and could have prognostic and therapeutic implications for cancer.

Eytan Zlotorynski

ORIGINAL ARTICLE Galanos, P. et al. Chronic p53-independent p21 expression causes genomic instability by deregulating replication licensing. Nat.Cell Biol. http://dx.doi.org/10.1038/ncb3378 (2016)

FURTHER READING Fragkos, M. et al. DNA

replication origin activation in space and time. Nat. Rev. Mol. Cell Biol. 16, 360-374 (2015) 